\title{
Indicators of Airway Secretion Weight in Mechanically Ventilated Subjects
}

\author{
George Ntoumenopoulos, Marc P Berry, Luigi Camporota, and Lawrence Lam
}

\begin{abstract}
BACKGROUND: Clinicians may use adventitious breath sounds on lung auscultation and a "sawtooth" pattern on the ventilator expiratory flow waveform as indicators of the need for chest physiotherapy for airway-secretion clearance in mechanically ventilated patients. This study seeks to identify potential clinical and novel indicators of the weight of airway secretions cleared from a single session of chest physiotherapy in mechanically ventilated subjects. METHODS: We recorded airway crackles using artificial airway acoustic sound monitoring and computerized lung-sound amplitude using artificial airway acoustic sound detection and compared them to standard clinical assessments in $\mathbf{7 1}$ mechanically ventilated subjects immediately prior to a single session of chest physiotherapy. Correlational analyses were undertaken between the weight of airway secretions obtained after the single session of chest physiotherapy as the dependent variable and novel assessments, clinical assessments, patient characteristics, and ventilator parameters as the independent variables. Multiple linear regression analyses were then used to determine the best model to predict the weight of airway secretions obtained from the single chest physiotherapy session. Data are reported as mean and median as appropriate. Significance was set at $P<.05$. RESULTS: 71 mechanically ventilated subjects were included for analysis. Statistically significant associations with the weight of airway secretions included the presence of a sawtooth waveform on expiration and the novel assessment of average airway crackles during inspiration. The best predictive model of the weight of airway secretions included the presence of the sawtooth waveform on expiration and ventilator tidal volume. CONCLUSIONS: Simple clinical assessments used in this study were able to independently predict the weight of airway secretions cleared during a single session of chest physiotherapy. The novel assessments used in this investigation did not add any further value. Key words: secretions; mechanical ventilation; physiotherapy; auscultation; computerized lung sound monitoring. [Respir Care 2019;64(11):1377-1386. () 2019 Daedalus Enterprises]
\end{abstract}

\section{Introduction}

Endotracheal intubation and mechanical ventilation impair secretion clearance and can lead to lung collapse, consolidation, and ventilator-associated pneumonia. ${ }^{1}$ Air-

Dr Ntoumenopoulos is affiliated with the Physiotherapy Department, St Vincent's Hospital, Sydney, Australia. Dr Berry is affiliated with the Physiotherapy Department, Hampshire Hospitals NHS Foundation Trust, Basingstoke, and North Hampshire Hospital, Hampshire, United Kingdom. Dr Camporota is affiliated with the Department of Adult Critical Care, Guy's and St Thomas' NHS Foundation Trust, King's Health Partners, London, United Kingdom. Dr Lam is affiliated with the Public Health Faculty of Health \& Graduate School of Health, University of Technology, Sydney, Australia.

The authors have disclosed no conflicts of interest way suctioning is the current standard of care for secretion clearance in the mechanically ventilated patient. ${ }^{2}$ Clinical indicators of the need for airway suctioning in mechanically ventilated patients currently include the presence of adventitious breath sounds centrally located on lung auscultation $^{3}$ and a "sawtooth" pattern on the ventilator expiratory flow waveform. ${ }^{4}$ These indicators can detect the presence of airway secretions $>0.5 \mathrm{~mL}$, obtained with

\footnotetext{
Correspondence: George Ntoumenopoulos PhD BAppSc (Physio), Physiotherapy Department, St Vincent's Hospital, 390 Darlinghurst Road, Darlinghurst, Sydney, New South Wales 2010, Australia. E-mail: georgentou@yahoo.com
}

DOI: $10.4187 /$ respcare.06437 
airway suctioning, with likelihoods ratios of 2.50 and 2.75, respectively. The likelihood ratio to detect airway secretions $>0.5 \mathrm{~mL}$ with airway suctioning increases to 14.7 when adventitious breath sounds and the sawtooth expiratory flow pattern are both present. ${ }^{4}$ However, the clinical impact of the $0.5-\mathrm{mL}$ secretion on patient outcomes is unknown. ${ }^{4}$ Chest physiotherapy in the mechanically ventilated patient can be used to assist with secretion clearance and may include techniques such as patient positioning (often head down), manual chest wall techniques (eg, chest wall percussion and vibrations), and manual/ventilator lung hyperinflation. ${ }^{5}$ Chest physiotherapy techniques can increase the weight of airway secretions cleared (on average $5 \mathrm{~g}$ ), which can result in improvements in respiratory compliance ${ }^{6}$ and re-expansion of a collapsed lung. ${ }^{7,8}$ Unfortunately, there are currently no reliable methods to predict the airway secretion wet-weight to be cleared with chest physiotherapy, making it unclear when airway suction alone is sufficient or when chest physiotherapy is indicated.

Computer-based acoustic technology provides objective and reproducible measurements of breath sounds in normal and in mechanically ventilated subjects with parenchymal lung diseases. ${ }^{9-13}$ This methodology may be able to standardize the detection of airway secretions in the mechanically ventilated patient. A commercial acoustic lung-imaging technology known as vibration response imaging (VRI) represents the unique distribution of breath sounds using a grayscale image. ${ }^{14}$ The dynamic lung image generated with VRI provides a continuous visualization of the characteristics of breath sounds along the complete breathing cycle. ${ }^{14}$ The TBAcare device (First Medica, Cusago, Italy) is connected to the ventilator circuit close to the endotracheal tube and generates an acoustic and visual signal with the automated detection of crackles when airway secretions are present. In a single-site study, TBAcare has been demonstrated to be a useful indicator of the need for airway suctioning as opposed to standard clinical assessments using lung auscultation. ${ }^{15}$ The TBAcare device may thus be another potential tool to gauge the presence of airway secretions in the mechanically ventilated patient.

The aims of this study were to determine whether standard clinical assessments, computerized lung sound monitoring, and artificial airway acoustic detection could reliably predict the weight of airway secretions cleared with a single session of chest physiotherapy.

\section{Methods}

\section{Study Site and Inclusion Criteria}

This study was undertaken in the Adult Intensive Care Department of the Guy's and St Thomas' NHS Foundation

\section{QUICK LOOK}

\section{Current knowledge}

Chest physiotherapy is routinely provided within intensive care for mechanically ventilated patients. However, there are no reliable methods to predict the weight of airway secretions present in a patient. Such a method may indicate whether airway suction alone is sufficient or chest physiotherapy is necessary.

\section{What this paper contributes to our knowledge}

Standard clinical assessments in the mechanically ventilated subject, including the presence of a sawtooth waveform on expiration and ventilator tidal volume, allow care providers to predict the weight of airway secretions cleared from a single session of chest physiotherapy. Novel computerized lung and airway sound monitoring was not associated with the weight of airway secretions cleared.

Trust in London. The study was approved by the Research and Development Department at Guy's and St Thomas' NHS Foundation Trust London, and ethical approval was obtained from the South-East London Ethics Committee (Ethics number: 09-39). Intubated and mechanically ventilated patients admitted between October 2011 and May 2012 were prospectively screened for study eligibility and enrollment. Study inclusion criteria included the planned assessment by a physiotherapist for the need for chest physiotherapy intervention with further details provided elsewhere. ${ }^{16} \mathrm{~A}$ physiotherapist is part of the multidisciplinary team in most ICUs. Physiotherapists use a comprehensive multisystem assessment that includes the respiratory, cardiovascular, neurological, and musculoskeletal systems to formulate individualized treatment plans that include respiratory and rehabilitation interventions. ${ }^{17}$ Informed consent was gained from the participants' next of kin to enroll in the study at the request of the Ethical Committee. Subjects were only studied on one occasion.

\section{Study Protocol}

On the day of the assessment, prior to the chest physiotherapy intervention, no endotracheal suction was performed within $2 \mathrm{~h}$ of the application of the research protocol. If endotracheal suction was considered clinically necessary during this period, then the protocol was delayed until no endotracheal suction was performed within $2 \mathrm{~h}$ of the application of the research protocol. 


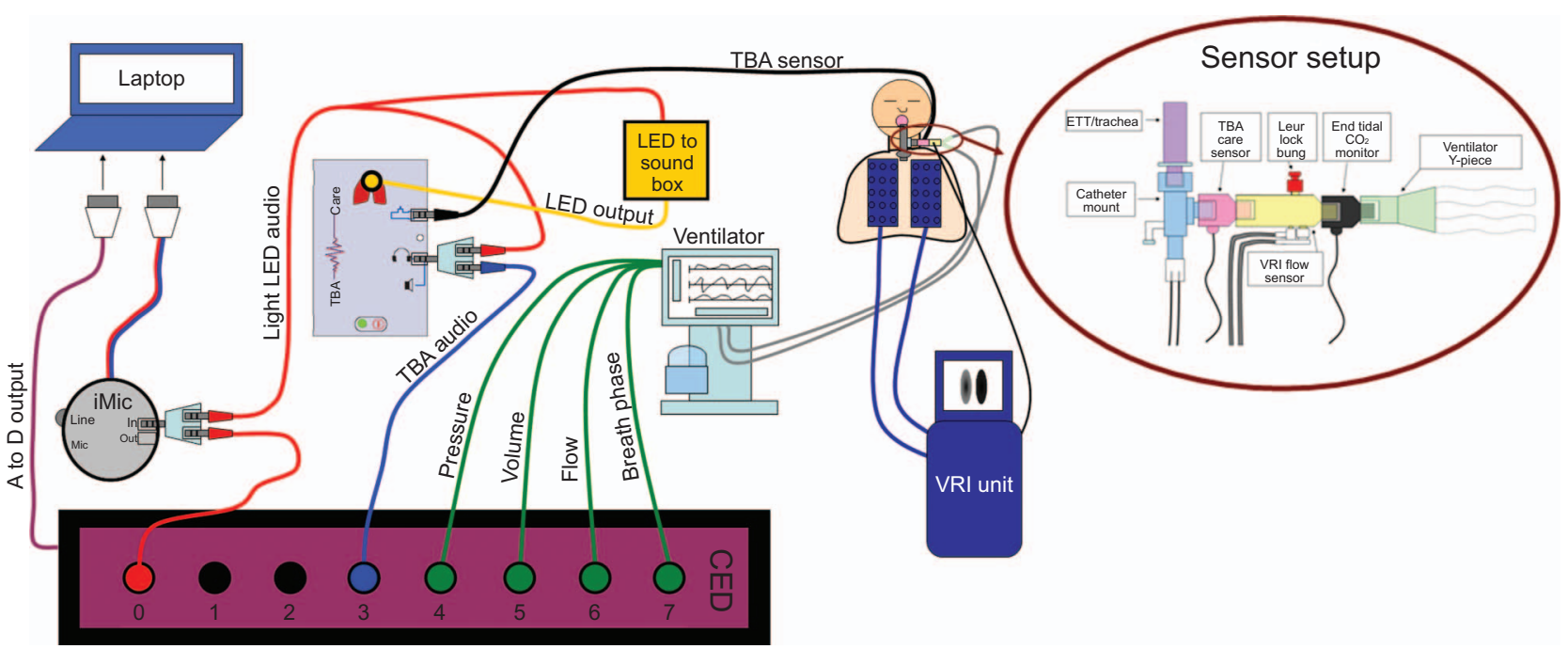

Fig. 1. Equipment setup.

\section{Device Preparation}

Prior to chest physiotherapy intervention, all subjects were established on the Viasys Avea ventilator (CareFusion, California). The ventilator visual display unit was connected to a personal computer via a VGA to USB capture device (Epiphan Systems, Ottawa, Canada) to record 30-s clips of standard ventilator waveforms (pressure, flow, and volume across time) and ventilator parameters, in particular, tidal volumes, breathing frequency, peak inspiratory flow, and peak expiratory flow (Fig. 1). These were recorded through dedicated software (Epiphan Screen Capture Tools, Epiphan Systems) associated with the device. Flow, volume, pressure, and breath phase analog ventilator waveforms were converted to digital waveforms with an analog-to-digital converter (Power 1401, CED, Cambridge, United Kingdom). In turn, these waveforms were recorded on a computer via dedicated software (Spike 2, Version 7, CED, Cambridge, United Kingdom). TBAcare analog signals were converted to digital waveforms via the aforementioned converter, and 2 signals were captured. First, a light sensor was used to capture the triggering of the TBAcare LED alarm, which in turn was converted to an analog audio signal. Second, the analog audio input was acquired from the TBAcare acoustic sensor placed proximal to the patient, between the ventilator circuit and the lateral connection of the closed suction circuit. Both signals were recorded continuously throughout the datacollection phase. The full equipment setup is shown in Figure 1.

The flow sensor of the VRI unit (VRIxv, Deep Breeze, Or-Akiva, Israel) was connected between the TBAcare sensor and the ventilator circuit. Subjects were positioned semirecumbent $\left(30-45^{\circ}\right.$ at the head of the bed), and V- array pads were adjusted as necessary until image quality was validated.

Ventilator flow, pressure, and VRI waveforms were synchronized with the image captured on the VRI display unit with an integrated flow sensor in situ (see sensor setup in Fig. 1). ${ }^{13,14}$

\section{VRI Recording Procedure}

The subject laid on 2 arrays of sensors or microphones similar to those used in digital stethoscopes (Deep Breeze). Acoustic signals were recorded during $20 \mathrm{~s}$ of acquisition time, followed by $40 \mathrm{~s}$ of processing time. A normalized dynamic image was displayed after each VRI recording, and the raw data were stored digitally on the device for later review and analysis. Recording quality was assessed according to predetermined criteria. ${ }^{13,14}$ Artifacts were easily identified, and recordings of poor quality were excluded. ${ }^{13,14}$

\section{Measurement Output}

Data collected with the VRI sensors during a 20-s recording were processed, and a grayscale video depicting the relative regional breath sound distribution of respiratory sounds was created. ${ }^{14}$ A sequential display of the images from $0.17 \mathrm{~s}$ of data were displayed $60 \mathrm{~s}$ after the start of the VRI recording. This generated a short video that demonstrated the changes that occurred in the distribution of vibration energy across the lung regions over time. ${ }^{18}$ VRI images can be processed to provide absolute vibration energy amplitude, relative acoustic factors, including regional vibration energy distribution, and parameters reflecting homogeneity of breath sound distribution, 
Table 1. Mean Value in Normal Lungs

\begin{tabular}{lc}
\hline \hline Vibration energy amplitude & $9 \pm 8\left(10^{6} \times\right.$ arbitrary units $)$ \\
Vibration energy distribution & \\
Right lung & $44 \pm 7 \%$ \\
Left lung & $56 \pm 7 \%$ \\
Homogeneity index & $86 \pm 6 \%$ \\
\hline
\end{tabular}

including the homogeneity index and apico-diaphragmatic ratio. Detailed information on the VRI filtering and image creation are described elsewhere. ${ }^{13,14}$ A normalized representative image frame at peak inspiratory flow is automatically selected by the VRI software. This image is quantified by the VRI software and presented as the percentage of weighted pixels in the 6 lung regions of interest: upper right; middle right; lower right; upper left; middle left; and lower left, up to a total of $100 \%$. For example, if airway secretions occluded the distal and or major airways and disrupted regional ventilation, then conceivably the measures of the distribution of regional ventilation, including vibration energy distribution, homogeneity index, and apico-diaphragmatic ratio, will be affected. ${ }^{14}$ In normal healthy individuals, using VRI provides very good reproducibility for total lung (intraclass correlation coefficient $=0.86$ ) and 6-zone assessment (intraclass correlation coefficient $=0.83$ ) of vibration energy amplitude. ${ }^{19}$ When compared to normal-appearing lungs (based on the chest radiograph findings), diseased lungs demonstrated significantly reduced vibration energy with significantly greater asymmetry between the lungs. ${ }^{18}$

The VRI device monitors absolute vibration energy amplitude as well as relative acoustic factors, such as regional vibration energy distribution, and parameters reflecting homogeneity of lung sound distribution (eg, homogeneity index and apico-diaphragmatic ratio). Mean ( \pm SD) VRI values in normal lungs in the supine position and are presented (Table 1).

\section{Schedule of Assessments and Intervention}

Prior to assessment, any condensate from the mechanical ventilator circuit or water traps was removed, oral secretions were suctioned, and the subglottic ports of the endotracheal tubes were aspirated. Airway suction circuit pressure was set at $-150 \mathrm{~mm} \mathrm{Hg}$, and the closed suction unit catheter was flushed with $5 \mathrm{~mL} 0.9 \%$ saline solution to remove residual secretions from the lumen. New suction tubing was connected, and 2 mucous specimen traps were inserted within the suction circuit to capture sputum. The primary trap was placed proximally to the patient, and a reserve trap was positioned distally to capture any secretions bypassing the primary trap. Prior to their insertion into the circuit, the dry traps were weighed on medical-grade

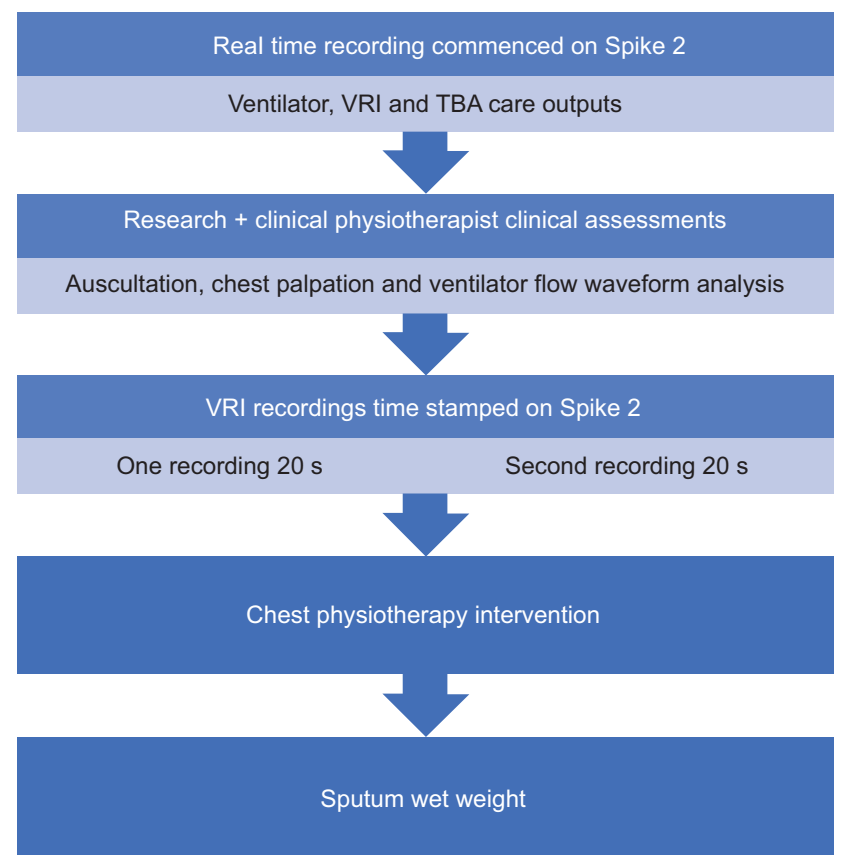

Fig. 2. Schedule of assessments.

scales (Traveler Scales, Ohaus, Parsippany, New Jersey), and their weights were recorded.

\section{Assessments, Intervention, and Outcome Measures}

At initial assessment, the real-time recording of all ventilator and TBAcare waveforms were commenced in Spike 2 (Fig. 2). Physiotherapists then began their clinical assessment of the need for secretion clearance based on local ICU physiotherapy treatment guidelines (ie, presence of adventitious sounds, presence of sawtooth pattern on the ventilator waveform, tactile chest wall/ventilator circuit fremitus). ${ }^{16}$ Lung auscultation was undertaken using a dual-limb stethoscope (Classic II SE Teaching Stethoscope, 3M Littmann, St Paul, Minnesota) to allow the ICU and research physiotherapists to independently auscultate the same lung regions at the same time. Six standard anterolateral lung areas (right and left upper, middle, and lower zones) were assessed with the subject in a supine, head-up, $30^{\circ}$ position. The ICU and research physiotherapists documented their auscultation findings separately on standardized data sheets, using standardized terminology for each lung area, blinded to each other's findings. They included information on the presence or absence of breath sounds, type of additional abnormal (ie, bronchial breathing) and adventitious breath sounds (ie, crackles, wheezes, or pleural rub), including the phase of respiration in which these sounds occurred. Bilateral chest wall palpation of the same 6 lung regions as auscultation was undertaken by the ICU and research physiotherapists to assess the presence of 
tactile fremitus. Anatomical areas were not marked out; hence, hand positioning was approximated as per clinical practice. The findings of the ICU and research clinicians were recorded, again independently of each other without knowledge of each other findings. Flow-time waveforms and ventilator parameters were recorded in real time for 30 s using a screen-capture device (Epiphan Screen Capture Tools, Epiphan Systems, Ottawa, Canada). The 30-s screen capture recording of ventilator flow-time waveforms was visually inspected for the sawtooth pattern by both the ICU and research physiotherapist. Once again, their assessments were documented independently, maintaining blinding. The chest radiograph was reviewed independently by both the clinical physiotherapist and the research physiotherapist. The research physiotherapist utilized a standardized assessment tool to report the radiological findings on the presence or absence of key pathologies, including atelectasis, consolidation, pleural effusion, pneumothorax, and pulmonary edema. The chest radiograph report from the radiologist was utilized to identify the key pathological findings of atelectasis, consolidation, pleural effusion, pneumothorax, and pulmonary edema reported in this study.

After the physical assessments, 2 separate 20-s periods of VRI imaging were recorded and time-marked in Spike 2. Once these assessments had been completed, the clinical physiotherapist carried out a single chest physiotherapy treatment as deemed appropriate, ensuring that at least 1 endotracheal suction pass was performed with the mucous trap connected; the research physiotherapist was not present during this intervention and remained blinded to the type of chest physiotherapy intervention used. Chest physiotherapy could have included combinations of normal saline lavage $(0.9 \% \mathrm{NaCl})$, postural drainage, manual chest-wall vibrations, manual-assisted cough, and ventilator/manual lung hyperinflations, depending on clinical indications as previously described. ${ }^{16,20}$ The chest physiotherapy interventions selected by the clinical physiotherapist were based on their initial clinical assessments and clinical suspicion for secretion presence, and hence the intervention varied on the basis of the perceived clinical needs of the patient at the time of assessment. Chest physiotherapy interventions were not standardized because the indications for specific interventions were unknown until the assessment by the physiotherapist had been undertaken. Hence, it would have been unethical to standardize a specific chest physiotherapy intervention. However, airway suctioning was to be undertaken to ensure the measurement of the weight of airway secretions. Chest physiotherapy was provided until the patient was deemed to be clear of any signs of secretion retention (eg, tactile fremitus on the chest wall, catheter mount, adventitious breath sounds such as wheezes and crackles on auscultation, sawtooth pattern on the expiratory flow waveform) that may have been present on initial assessment or until there were minimal secretions obtained on airway suctioning. Saline instillation into the artificial airway was used as clinically indicated, and the volume of saline used was documented. When the chest physiotherapy treatment was complete, the closed-suction catheter was flushed with $5 \mathrm{~mL}$ sterile $0.9 \%$ normal saline solution, and both mucous traps were then re-weighed. From this, the weight of the dry traps and the $5 \mathrm{~mL} 0.9 \%$ normal saline flush were subtracted to calculate the sputum wet-weight obtained from the intervention. For the purposes of this trial, only the weight of airway secretions obtained as a result of the chest physiotherapy intervention was reported and used as an indicator of the presence of airway secretions in the subject at the time of the study. The full schedule of the assessments and intervention can be seen in Figure 2.

\section{Statistical Analysis}

This was an exploratory study with no a priori knowledge of the effect size, so we could not conduct a proper sample-size calculation. Based on an assumption of using multiple regression analysis as the analytical approach with a medium effect size of $\mathrm{f}^{2}=0.35,80 \%$ power of study, a type- 1 error rate of $5 \%$, and at least 2 predictors in the model a sample size of at least 68 subjects would be required.

Data are presented using descriptive statistics as pretreatment mean (SD) and median (interquartile range [IQR]) as appropriate. VRI and TBAcare data were assessed on 2 separate occasions before chest physiotherapy, and the mean of the data are presented. Data analysis was performed with statistic software (SPSS version 11.5, IBM, Armonk, New York) with expert statistical support. Initial correlation analyses were undertaken to explore the relationship between the weight of airway secretions removed to the pretreatment findings of lung sound amplitude on VRI (ie, vibration energy amplitude), crackles identified with TBAcare, clinical assessment findings (lung auscultation, chest wall, and tactile fremitus on the ventilator tubing), and the presence of a sawtooth pattern on ventilator flow waveforms as detected independently by the research physiotherapist. The number of clinical and novel assessments included in the analyses were restricted due to the sample size and collapsed down to 9 variables on the basis of clinical experience: vibration energy amplitude (total left and right lung), presence of any chest wall fremitus (inspiratory or expiratory), presence of ventilator tubing fremitus (inspiratory), presence of ventilator tubing fremitus (expiratory), presence of sawtooth ventilator waveform on expiration, presence of any adventitious breath sounds (from any 6 lung regions), ventilator flow bias (inspiratory/expiratory), average TBAcare value (inspiratory), and average TBAcare value (expiratory). Multiple 
linear regression analyses were then used to determine the best model to predict the weight of airway secretions obtained from the single chest physiotherapy session. For testing of hypotheses, a type-1 error of 5\% was employed. As an exploratory study, no interaction terms of predictor variables were attempted in the regression analyses.

\section{Results}

A total of 71 mechanically ventilated subjects were studied. Demographics and clinical characteristics are provided in Table 2. Most subjects were male (59\%), with a mean (SD) admission APACHE II score of 19.1 (5.6), and most were mechanically ventilated $(75 \%$ with endotracheal tubes, $25 \%$ tracheostomy tubes) with a median of 8 and the IQR of (7-9) $\mathrm{mm}$. Heated humidifiers were used with 45 subjects, and heat and moisture exchanger filters were used with the remaining 26 subjects. Most of the subjects were on spontaneous or assisted modes of ventilation (Table 2). Most subjects were admitted to intensive care due to medical conditions (ie, 52 medical admissions, 19 surgical admissions), and 15 subjects also had a past history of COPD or asthma (Table 2).

Subjects were studied a mean (SD) of 202 (194) h after intubation. During the time of clinical assessment, the subjects had a median (range) PEEP of $5(5-10) \mathrm{cm} \mathrm{H}_{2} \mathrm{O}$, mean (SD) $\mathrm{F}_{\mathrm{IO}_{2}}$ of $31.4 \%$ (7.4), with a mean (SD) tidal volume of 439 (150) $\mathrm{mL}$, and most of the subjects were lightly sedated (median [range] Richmond Agitation-Sedation Scale score of -1 [ -5 to 2]); only one subject required neuromuscular blocking agents. In all of the subjects, the hemodynamic parameters at the time of assessment were within normal range (Table 2).

The average $\mathrm{P}_{\mathrm{aO}_{2}} / \mathrm{F}_{\mathrm{IO}_{2}}$ ratio was $247 \mathrm{~mm}$ Hg. Chest radiography on the day of assessment showed subjects had bilateral atelectasis $(n=11)$, unilateral left-lung atelectasis $(n=24)$, or right-lung atelectasis $(n=18)$. The median (IQR) weight of airway secretions obtained after a single session of chest physiotherapy was 8 gm (IQR [414]) $(n=69)$. Physiotherapists suspected the presence of airway secretions in the subjects in this study as they used a median (IQR) of 4 (2-6) chest physiotherapy techniques, with the most frequently used techniques of closed suctioning, chest wall vibrations, postural drainage with trendelenburg positioning, and saline lavage (Fig. 3), with a mean (SD) treatment time of 19.6 (10.2) min.

\section{Standard Clinical and Novel Assessments}

The summary data of the clinical and novel assessments prior to chest physiotherapy are presented in Table 3. Prior to chest physiotherapy, most subjects $(94-100 \%$ of subjects) had normal breath sounds present on standard lung auscultation.
Table 2. Subject Demographics and Clinical Characteristics

\begin{tabular}{|c|c|}
\hline Age, y & $61(17)$ \\
\hline Male, $n(\%)$ & $42(59)$ \\
\hline $\begin{array}{l}\text { Hours mechanically ventilated prior to } \\
\text { assessment, } \mathrm{h}\end{array}$ & $202(194)$ \\
\hline APACHE II score at admission & $19.1(5.6)$ \\
\hline SOFA score at admission & $7.9(3.3)$ \\
\hline $\begin{array}{l}\text { SOFA score on day of assessment } \\
\quad(n=70 \text { subjects })\end{array}$ & $6.2(3.4)$ \\
\hline Medical admission, $n(\%)$ & $52(73)$ \\
\hline Surgical admission, $n(\%)$ & $19(27)$ \\
\hline COPD/asthma, $n^{*}(\%)$ & $15(21)$ \\
\hline Weight, $\mathrm{kg}$ & $77(19)$ \\
\hline \multicolumn{2}{|c|}{ Airway/mechanical ventilation/cardiorespiratory } \\
\hline \multicolumn{2}{|c|}{ Type of artificial airway, $n(\%)$} \\
\hline Orotracheal tube & $53(75)$ \\
\hline Tracheostomy & $18(25)$ \\
\hline $\begin{array}{l}\text { Median inner-airway diameter, } \mathrm{mm} \\
\text { (interquartile range) }\end{array}$ & $8(7-9)$ \\
\hline \multicolumn{2}{|l|}{ Humidification, $n(\%)$} \\
\hline Heated & $45(63)$ \\
\hline Heat and moisture exchanger & $26(37)$ \\
\hline \multicolumn{2}{|l|}{ Ventilation mode, $n(\%)$} \\
\hline CPAP alone & $16(23)$ \\
\hline $\mathrm{CPAP}+$ pressure support & $46(65)$ \\
\hline Pressure SIMV & $1(2)$ \\
\hline PRVC SIMV & $5(7)$ \\
\hline Bilevel positive airway pressure & $2(3)$ \\
\hline $\mathrm{F}_{\mathrm{IO}_{2}}$ & $0.31(7.4)$ \\
\hline Median PEEP, $\mathrm{cm} \mathrm{H}_{2} \mathrm{O}$ (range) & $5(5-10)$ \\
\hline Peak inspiratory flow, L/min & $53.9(15.6)$ \\
\hline Peak expiratory flow, L/min & $35.9(10.5)$ \\
\hline Tidal volume, $\mathrm{mL}$ & $439(150)$ \\
\hline Frequency, total (breaths/min) & $23(8.8)$ \\
\hline \multicolumn{2}{|l|}{ Respiratory and hemodynamic parameters } \\
\hline Oxygen saturations, $\mathrm{S}_{\mathrm{pO}_{2}}$ & $94.8(2.4)$ \\
\hline Median RASS (range) & $-1(-5$ to 2$)$ \\
\hline Systolic blood pressure, $\mathrm{mm} \mathrm{Hg}$ & $126(23)$ \\
\hline Diastolic blood pressure, $\mathrm{mm} \mathrm{Hg}$ & $60(10)$ \\
\hline Heart rate, beats/min & $98(19)$ \\
\hline \multicolumn{2}{|l|}{$\begin{array}{l}\text { Arterial blood gas (pre-physiotherapy } \\
\text { assessment) }\end{array}$} \\
\hline $\mathrm{pH}$ & $7.41(0.06)$ \\
\hline $\mathrm{P}_{\mathrm{aO}_{2}}, \mathrm{~mm} \mathrm{Hg}$ & $78.46(12.52)$ \\
\hline $\mathrm{P}_{\mathrm{aCO}_{2}}, \mathrm{~mm} \mathrm{Hg}$ & $45.15(8.10)$ \\
\hline $\mathrm{HCO}_{3}^{-}$ & $26.86(3.28)$ \\
\hline Median base excess (range) & $2.5(-5.5$ to 10.2$)$ \% \\
\hline $\begin{array}{l}N=71 \text { subjects. Values are mean (SD) or frequency } \\
* \text { COPD or asthma in past medical history. } \\
\text { APACHE II = Acute Physiology and Chronic Healt } \\
\text { SOFA = Sequential Organ Failure Assessment score } \\
\text { SIMV = synchronized intermittent mandatory mecha } \\
\text { PRVC = pressure-regulated volume control ventilat } \\
\text { RASS = Richmond Agitation Sedation Scale }\end{array}$ & $\begin{array}{l}\text { therwise noted. } \\
\text { II } \\
\text { ion }\end{array}$ \\
\hline
\end{tabular}

The most common adventitious breath sounds on lung auscultation were inspiratory crackles in the left lower zones (34\% of subjects) followed by inspiratory wheeze in 


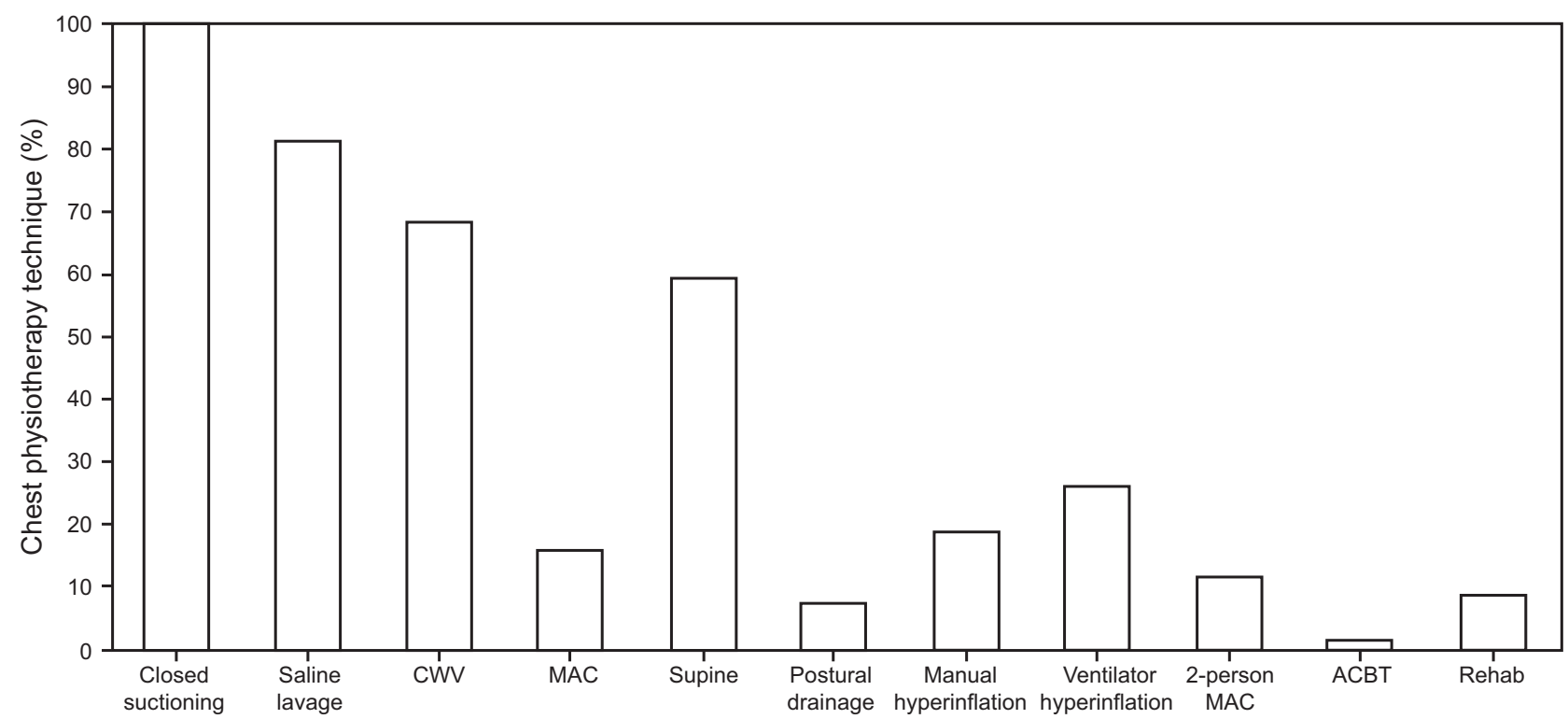

Fig. 3. Chest physiotherapy interventions. $\mathrm{CWV}=$ chest wall vibrations; $\mathrm{MAC}=$ manually assisted cough, $\mathrm{ACBT}=\mathrm{Active}$ cycle breathing technique.

the right middle zone (24\%) and the left upper zones (23\%), inspiratory wheeze in the left middle zone $(23 \%)$, inspiratory wheeze in the right lower zone $(21 \%)$, inspiratory wheeze in right upper zone (20\%), inspiratory crackles in the left middle zone (20\%), and expiratory crackles in the left middle zone (20\%).

Tactile chest wall fremitus was more frequently present bilaterally in the upper zones (31\%) followed by the right middle zone (23\%). Tactile ventilator tubing fremitus was also more evident during inspiration. The sawtooth pattern on the ventilator flow waveform was found in $44 \%$ of subjects and was predominantly present during expiration.

The mean (SD) vibration energy distribution percentage values for the left lung were $42.2 \%$ (21.3), which was lower than the expected mean (SD) vibration energy distribution percentage values from the normal healthy left lung, ie, $56 \%$ (7.0). The mean (SD) vibration energy distribution percentage values from the right lung were $42.1 \%$ (21.3), which was similar to the expected mean (SD) vibration energy distribution percentage values for a normal healthy right lung, ie, $44 \%$ (7.0). Similarly, the mean (SD) vibration energy amplitudes $\left(\times 10^{6} \mathrm{AU}\right)$ for the left lung were lower than those for the right lung at 7.8 (24.9) and 8.7 (26.4), respectively; however, the combined values for both lungs were nearly double the expected normal values of normal healthy lungs at 9.0 (8.0). The mean (SD) homogeneity index as an indicator of distribution of breath sounds between the lungs was $67.5 \%$ (30.9), which was lower than the expected normal value of $86.0 \%$ (6.0). The TBAcare mean (SD) values as indicators of the presence of airway crackles were higher during inspiration, 1.89 (3.41) than expiration 1.45 (5.84).

\section{Univariate Associations With the Weight of Airway Secretions}

There were statistically significant positive univariate associations in Table 4 between the weight of airway secretions and the presence of the sawtooth waveform on expiration $\left(\mathrm{t}_{67}=-2.69, P=.01\right)$ and the average number of crackles during inspiration detected with the TBAcare device $(\mathrm{r}=0.30, P=.02)$.

There was a trend between the weight of airway secretions and the average number of crackles detected with the TBAcare during expiration $(\mathrm{r}=0.25, P=.05)$. There was no association between the weight of airway secretions and the detection of fremitus on the ventilator tubing, ventilator flow bias, the presence of any adventitious sounds on lung auscultation, the presence of chest wall fremitus, or the vibration energy amplitude (total from both lungs) from the VRI device.

\section{Multiple Regression Model of Predictors of Secretion Wet-Weight}

Multiple linear regression models were used to investigate the potential predictors of the weight of airway secretions (Table 5). The best predictive model with a $\mathrm{r}^{2}$ of 0.17 included the variables of sawtooth waveform pattern on expiration $(\beta=4.46, P=.01)$ and the ventilator tidal volume $(\beta=0.01, P=.02)$.

\section{Discussion}

There were statistically significant positive univariate associations between the weight of airway secretions from 
Table 3. Clinical and Novel Assessments Prior to Chest Physiotherapy

\begin{tabular}{|c|c|c|}
\hline Assessments & Left Lung & Right Lung \\
\hline \multicolumn{3}{|l|}{ Normal breath sounds } \\
\hline Upper zone & $71(100)$ & $71(100)$ \\
\hline Middle zone & $70(99)$ & $70(99)$ \\
\hline Lower zone & $67(94)$ & $67(94)$ \\
\hline \multicolumn{3}{|l|}{$\begin{array}{l}\text { Adventitious breath sounds on lung auscultation } \\
\text { and fremitus }\end{array}$} \\
\hline \multicolumn{3}{|l|}{ Inspiration wheeze } \\
\hline Upper zone & $16(23)$ & $14(20)$ \\
\hline Middle zone & $16(23)$ & $17(24)$ \\
\hline Lower zone & $9(13)$ & $15(21)$ \\
\hline \multicolumn{3}{|l|}{ Inspiration crackles } \\
\hline Upper zone & $8(11)$ & $10(14)$ \\
\hline Middle zone & $14(20)$ & $13(18)$ \\
\hline Lower zone & $24(34)$ & $17(24)$ \\
\hline \multicolumn{3}{|l|}{ Expiration wheeze } \\
\hline Upper zone & $6(9)$ & $7(10)$ \\
\hline Middle zone & $6(9)$ & $7(10)$ \\
\hline Lower zone & $6(9)$ & $5(7)$ \\
\hline \multicolumn{3}{|l|}{ Expiration crackles } \\
\hline Upper zone & $11(16)$ & $13(18)$ \\
\hline Middle zone & $9(13)$ & $14(20)$ \\
\hline Lower zone & $8(11)$ & $9(13)$ \\
\hline \multicolumn{3}{|l|}{ Bronchial breathing } \\
\hline Upper zone & $0(0)$ & $0(0)$ \\
\hline Middle zone & $1(1)$ & $1(1)$ \\
\hline Lower zone & $2(3)$ & $6(9)$ \\
\hline \multicolumn{3}{|l|}{ Pleural rub } \\
\hline Upper zone & $1(1)$ & $0(0)$ \\
\hline Middle zone & $1(1)$ & $1(1)$ \\
\hline Lower zone & $1(1)$ & $2(3)$ \\
\hline \multicolumn{3}{|l|}{ Chest wall and ventilator tubing fremitus } \\
\hline Upper zone & $22(31)$ & $22(31)$ \\
\hline Middle zone & $9(13)$ & $16(23)$ \\
\hline Lower zone & $7(6)$ & $3(4)$ \\
\hline \multicolumn{3}{|l|}{ Airway/catheter mount fremitus } \\
\hline On inspiration & \multicolumn{2}{|c|}{$35(49)$} \\
\hline On expiration & \multicolumn{2}{|c|}{$6(9)$} \\
\hline \multicolumn{3}{|l|}{ Clinician-detected sawtooth flow pattern } \\
\hline On inspiration & \multicolumn{2}{|c|}{$9(12.7)$} \\
\hline On expiration & \multicolumn{2}{|c|}{$22(31.0)$} \\
\hline VRI-detected sawtooth flow pattern & \multicolumn{2}{|c|}{$15(21)$} \\
\hline \multicolumn{3}{|l|}{ VED, \%* } \\
\hline Upper zone (UZ) & $11.8(7.9)$ & $13.2(6.2)$ \\
\hline Middle zone (MZ) & $17.9(9.0)$ & $17.7(8.8)$ \\
\hline Lower zone (LZ) & $12.6(8.3)$ & $13.2(9.4)$ \\
\hline Upper quadrant (UQ) & $20.5(12.2)$ & $19.8(11.2)$ \\
\hline Lower quadrant (LQ) & $21.4(11.9)$ & $21.9(13.3)$ \\
\hline Total (for $\mathrm{UZ}+\mathrm{MZ}+\mathrm{LZ}$ and for $\mathrm{UQ}+\mathrm{LQ}$ ) & $42.2(21.3)$ & $42.1(21.3)$ \\
\hline \multicolumn{3}{|l|}{$\operatorname{VEA}\left(10^{6} \times \mathrm{AU}\right) \dagger$} \\
\hline Upper quadrant & $3.7(12.8)$ & $2.9(8.7)$ \\
\hline Lower quadrant & $4.1(12.6)$ & $5.8(18.5)$ \\
\hline Total & $7.8(24.9)$ & $8.7(26.4)$ \\
\hline Homogeneity index, \%末 & \multicolumn{2}{|c|}{$67.5(30.9)$} \\
\hline TBAcare & & \\
\hline Inspiration & & .41) \\
\hline Expiration & & .84) \\
\hline $\begin{array}{l}N=71 \text { subjects. Assessments are shown as either freq } \\
* \text { Normal VED (SD) values: left lung, } 56(7 \%) \text {; right } 1 \\
\dagger \text { Normal VEA }\left(10^{6} \times \mathrm{AU}\right) \text { total both lungs }=9(8) . \\
\text { † Normal homogeneity index value }=86(6 \%) . \\
\text { VRI }=\text { vibration response imaging } \\
\text { VED }=\text { vibration energy distribution } \\
\text { VEA = vibration energy amplitude } \\
\text { AU }=\text { arbitrary units }\end{array}$ & $\begin{array}{l}y(\%) \text { or mean } \\
44(7 \%) \text {. }\end{array}$ & \\
\hline
\end{tabular}

Table 4. Unadjusted Associations Between Possible Predictors of Airway Secretion Weight

\begin{tabular}{llc}
\hline \hline \multirow{2}{*}{\multicolumn{1}{c}{ Possible Predictors }} & \multicolumn{2}{c}{$\begin{array}{c}\text { Sputum } \\
\text { Weight, } \mathrm{mL}\end{array}$} \\
\cline { 2 - 3 } & Association & $P$ \\
\hline VEA (total left and right lungs) & $\mathrm{r}=0.10$ & .40 \\
Chest wall fremitus* & $\mathrm{r}=0.14$ & .36 \\
Catheter mount fremitus (inspiration)* & $\mathrm{t}_{67}=-1.00$ & .42 \\
Catheter mount fremitus (expiration)* & $\mathrm{t}_{67}=0.50$ & .72 \\
Sawtooth expiration* & $\mathrm{t}_{67}=-2.69$ & .01 \\
Any adventitious breath sounds* & $\mathrm{r}=0.13$ & .36 \\
Flowrate bias & $\mathrm{r}=-0.12$ & .33 \\
TBAInspiration (average) & $\mathrm{r}=0.30$ & .02 \\
TBAExpiration (average) & $\mathrm{r}=0.25$ & .05 \\
& & \\
\hline$N=69$ subjects. & & \\
$*$ Indicates all assessments were made by the research physiotherapist (blinded to primary \\
outcome measure secretion weight). \\
VEA = vibration energy amplitude \\
TBAcare = crackle detector
\end{tabular}

Table 5. Results From the Final Model of the Multiple Linear Regression Analyses on Weight of Airway Secretions

\begin{tabular}{cccc}
\hline \hline Variables & $\beta$ & $\begin{array}{c}\text { Standard } \\
\text { Error } \\
\text { of } \beta\end{array}$ & Results \\
\hline $\begin{array}{c}\text { Sawtooth waveform pattern } \\
\text { on expiration }\end{array}$ & 4.46 & 1.56 & $\mathrm{t}=2.86(P=.01)$ \\
Ventilator tidal volume & 0.01 & 0.06 & $\mathrm{t}=2.24(P=.02)$ \\
\hline
\end{tabular}

a single chest physiotherapy intervention and clinical detection of a sawtooth waveform on expiration and the mean number of inspiratory crackles detected with the TBAcare device. The multiple linear regression model demonstrated that the only statistically significant predictors of the weight of airway secretions cleared from a single session of chest physiotherapy were the research physiotherapists' detection of sawtooth waveform pattern on expiration and ventilator tidal volume.

Previous research has demonstrated that the combined presence of adventitious breath sounds on auscultation and the presence of the sawtooth pattern on the expiratory flow waveform is significantly associated with $0.5 \mathrm{~mL}$ of airway secretions obtained with airway suctioning alone. ${ }^{4}$ Our study has demonstrated that using clinical assessments such as the detection of the sawtooth waveform and ventilator tidal volume are better than novel computerized assessments at judging the weight of airway secretions produced by a single session of chest physiotherapy.

VRI has been previously validated to identify various pleural and parenchymal lung diseases, and to detect changes in regional lung ventilation with different me- 
chanical ventilation strategies. ${ }^{21-26}$ However, our investigation failed to demonstrate any independent associations between the various VRI measures and the weight of airway secretions cleared as a result of a single session of chest physiotherapy. In contrast, the only previous study that used VRI prior to chest physiotherapy demonstrated an increased lung sound amplitude (ie, vibration energy amplitude) compared with normal values. In addition, because they demonstrated a 2-fold reduction in the lung sound amplitude following chest physiotherapy for secretion clearance in the mechanically ventilated patient, ${ }^{20}$ it was postulated that the increased airway secretion weight may have been the cause of the increased lung sound amplitude. Unfortunately, the investigators did not report on the clinical assessment findings before or after chest physiotherapy, nor did they quantify the weight of airway secretions removed with the chest physiotherapy interventions. The significant positive univariate association between TBAcare signal and the weight of airway secretions did not remain significant in the multiple linear regression model. A previous trial with the TBAcare device demonstrated a reduced need for airway suctioning when compared with standard clinical assessment. ${ }^{15}$ The authors postulated the TBAcare device to be a more appropriate indicator of the need for airway suctioning, however there was no impact on patient outcomes. We hypothesize that airway secretions in our subjects may be located more distal from the artificial airway (where the TBAcare device was located), which may reduce the sensitivity of the device to detect the weight of airway secretions in the distal airways. Further validation is required in an intubated and ventilated animal model with known presence of a specified weight, location, and viscosity of airway secretions to define and establish the valid indicators (both clinical and novel) of the need for interventions such as chest physiotherapy for secretion clearance. ${ }^{27}$

There are predominantly only low levels of evidence indicating a link between the presence of airway secretions and significant pulmonary complications in the mechanically ventilated patient. ${ }^{28-30}$ In a large, prospective, observational trial of 955 mechanically ventilated subjects, ${ }^{31}$ $8 \%$ of subjects developed severe hypoxemia, with atelectasis due to mucous plugging the second most common cause $(28 \%)$. More recently, a large, multi-center, point prevalence study reported mucous plugging events to occur in $2.7 \%$ of mechanically ventilated subjects over a single 24 -h period. ${ }^{5}$ Further studies are warranted to explore the definitions of the increased burden of secretions in the mechanically ventilated subject, such as mucous plugging events (eg, obstructive ventilator flow waveform patterns, inability to deliver mechanical ventilation with a requirement for manual lung ventilation ${ }^{5}$ ), the weight of airway secretions obtained throughout the 24-h day, and the impact on patient outcomes.
Investigations are required to prospectively evaluate the clinical indicators of the presence of airway secretions in the mechanically ventilated patient with an accepted standard comparator. Then the prevalence and incidence of an increasing presence of airway secretions and the impact on major patient outcomes may be further investigated. This will drive the further investigation of interventions such as chest physiotherapy to alleviate the impact of an increased secretion burden and additional exploration of the effect on key patient outcomes such as lobar collapse, pneumonia, and time on mechanical ventilation.

There are several limitations with this observational trial. Primarily this was a single-site study with small patient numbers, which limits the generalizability of our findings. We also relied on the ability of a single chest physiotherapy intervention to clear airway secretions as a surrogate marker of the burden of secretions at the time of clinical assessment. The effectiveness of this single chest physiotherapy intervention to estimate the weight of airway secretions is unclear without a true accepted standard to predict the presence of airway secretions. This investigation thus may have included subjects with other conditions such as asthma, pulmonary edema, or other pathologies ${ }^{26}$ that may mimic these breath sounds and novel assessment findings. We did, however, only enroll subjects in whom physiotherapy clinicians suspected the presence of airway secretions as an indicator for chest physiotherapy, but these subjects may have had other or co-existing pulmonary and cardiac conditions. Large animal models may provide the ideal scenario to validate the methods used for the clinical detection of secretion presence.

\section{Conclusion}

Routine clinical assessments used in this study, including the presence of the sawtooth waveform on expiration and ventilator tidal volume, were able to independently predict the weight of airway secretions cleared by a single session of chest physiotherapy.

\section{REFERENCES}

1. Konrad F, Schreiber T, Brecht-Kraus D, Georgieff M. Mucociliary transport in ICU patients. Chest 1994;105(1):237-241.

2. Branson RD. Secretion management in the mechanically ventilated patient. Respir Care 2007;52(10):1328-1342.

3. Brooks D, Thomas J. Interrater reliability of auscultation of breath sounds among physical therapists. Phys Ther 1995;75(12):1082-1088.

4. Guglielminotti J, Alzieu M, Maury E, Guidet B, Offenstadt G. Bedside detection of retained tracheobronchial secretions in patients receiving mechanical ventilation: is it time for tracheal suctioning? Chest 2000;118(4):1095-1099.

5. Ntoumenopoulos G, Hammond N, Watts NR, Thompson K, Hanlon G, Paratz JD, et al. Secretion clearance strategies in Australian and 
New Zealand intensive care units. Aust Crit Care 2018;31(4):191196.

6. Berney S, Denehy L, Pretto J. Head-down tilt and manual hyperinflation enhance sputum clearance in patients who are intubated and ventilated. Aust J Physiother 2004;50(1):9-14.

7. Stiller K, Geake T, Taylor J, Grant R, Hall B. Acute lobar atelectasis: a comparison of two chest physiotherapy regimens. Chest 1990; 98(6): 1336-1340.

8. Stiller KJS, Grant R, Geale T, Taylor J, Hall B. Acute lobar atelectasis: a comparison of five physiotherapy regimens. Physio Theory Practice 1996;12:197-209.

9. Ploy-Song-Sang Y, Martin RR, Ross WR, Loudon RG, Macklem PT. Breath sounds and regional ventilation. Am Rev Respir Dis 1977;116(2):187-199.

10. Murphy RL, Jr., Holford SK, Knowler WC. Visual lung-sound characterization by time-expanded wave-form analysis. New Engl J Med 1977;296(17):968-971.

11. Gavriely N, Cugell DW. Airflow effects on amplitude and spectral content of normal breath sounds. J Appl Physiol (1985) 1996;80(1): 5-13.

12. Gonzalez-Camarena R, Charleston-Villalobos S, Angeles-Olguin A, Aljama-Corrales T. Imaging the thoracic distribution of normal breath sounds. Methods Inf Med 2010;49(5):443-447.

13. Dellinger RP, Parrillo JE, Kushnir A, Rossi M, Kushnir I. Dynamic visualization of lung sounds with a vibration response device: a case series. Respiration 2008;75(1):60-72.

14. Dellinger RP, Jean S, Cinel I, Tay C, Rajanala S, Glickman YA, Parrillo JE. Regional distribution of acoustic-based lung vibration as a function of mechanical ventilation mode. Crit Care 2007;11(1): R26.

15. Lucchini A, Zanella A, Bellani G, Gariboldi R, Foti G, Pesenti A, et al. Tracheal secretion management in the mechanically ventilated patient: comparison of standard assessment and an acoustic secretion detector. Respir Care 2011;56(5):596-603

16. Berry MP, Marti JD, Ntoumenopoulos G. Inter-rater agreement of auscultation, palpable fremitus, and ventilator waveform sawtooth patterns between clinicians. Respir Care 2016;61(10):1374-1383.

17. Berney S, Haines K, Denehy L. Physiotherapy in critical care in Australia. Cardiopulm Phys Ther J 2012;23(1):19-25.

18. Jean S, Cinel I, Tay C, Parrillo JE, Dellinger RP. Assessment of asymmetric lung disease in intensive care unit patients using vibration response imaging. Anesth Analg 2008;107(4):1243-1247.
19. Maher TM, Gat M, Allen D, Devaraj A, Wells AU, Geddes DM. Reproducibility of dynamically represented acoustic lung images from healthy individuals. Thorax 2008;63(6):542-548.

20. Ntoumenopoulos G, Glickman Y. Computerised lung sound monitoring to assess effectiveness of chest physiotherapy and secretion removal: a feasibility study. Physiotherapy 2012;98(3):250-255.

21. Shi C, Boehme S, Bentley AH, Hartmann EK, Klein KU, Bodenstein $\mathrm{M}$, et al. Assessment of regional ventilation distribution: comparison of vibration response imaging (VRI) with electrical impedance tomography (EIT). PloS One 2014;9(1):e86638.

22. Anantham D, Herth FJ, Majid A, Michaud G, Ernst A. Vibration response imaging in the detection of pleural effusions: a feasibility study. Respiration 2009;77(2):166-172.

23. Bing D, Jian K, Long-feng S, Wei T, Hong-wen Z. Vibration response imaging: a novel noninvasive tool for evaluating the initial therapeutic effect of noninvasive positive pressure ventilation in patients with acute exacerbation of chronic obstructive pulmonary disease. Respir Res 2012;13:65.

24. Comce F, Bingol Z, Kiyan E, Tanju S, Toker A, Cagatay P, et al. Vibration-response imaging versus quantitative perfusion scintigraphy in the selection of patients for lung-resection surgery. Respir Care 2011;56(12):1936-1941.

25. Lev S, Glickman YA, Kagan I, Dahan D, Cohen J, Grinev M, et al. Changes in regional distribution of lung sounds as a function of positive end-expiratory pressure. Crit Care 2009;13(3):R66.

26. Lev S, Glickman YA, Kagan I, Shapiro M, Moreh-Rahav O, Dahan $\mathrm{D}$, et al. Computerized lung acoustic monitoring can help to differentiate between various chest radiographic densities in critically ill patients. Respiration 2010;80(6):509-516.

27. Comaru T, Fiori HH, Fiori RM, Padoim P, Stivanin JB, da Silva VD. Experimental model of atelectasis in newborn piglets. Respiratory care 2014;59(1):121-126.

28. Butala BP, Shah VR, Bhosale GP. Acute hypoxaemia due to intraoperative lung collapse after repositioning the patient. Indian J Anaesth 2011;55(4):395-398.

29. Zamanian M, Marini JJ. Pressure-flow signatures of central-airway mucus plugging. Crit Care Med 2006;34(1):223-226.

30. Shields JA, Nelson CM. Acute hypoxemia after repositioning of patient: a case report. AANA J 2004;72(3):207-210.

31. Mahmood NA, Chaudry FA, Azam H, Ali MI, Khan MA. Frequency of hypoxic events in patients on a mechanical ventilator. Int J Crit Illn Inj Sci 2013;3(2):124-129. 